\title{
Developing an endoscopic mucosal resection service in a district general hospital
}

\author{
Chris A Lamb, ${ }^{1}$ Jamie A Barbour ${ }^{2}$
}

\begin{abstract}
${ }^{1}$ Institute of Cellular Medicine, Newcastle University, The Medical School, Newcastle upon Tyne, UK

2Department of Gastroenterology, Gateshead Health NHS Foundation Trust, Queen Elizabeth Hospital, Gateshead, UK
\end{abstract}

\section{Correspondence to}

Dr Jamie A Barbour, Department of Gastroenterology, Gateshead Health NHS Foundation Trust, Queen Elizabeth Hospital, Sherriff Hill, Gateshead NE9 6SX, UK;

jamie.barbour@ghnt.nhs.uk

Received 21 June 2012 Revised 12 July 2012 Accepted 13 July 2012 Published Online First 11 August 2012

\begin{abstract}
Objective To describe the implementation of a formal single-operator led endoscopic mucosal resection (EMR) service in a district general hospital, and the effect on patient outcome of this service development.

Design Prospective audit during initiation and subsequent development of EMR service.

Setting District general hospital.

Patients All patients referred to EMR service between 1 January 2008 and 31 December 2011.

Interventions Nil in addition to clinical care. Main outcomes measured The number of EMRs per year including polyp size and histology, recurrence of polyp tissue at 3 months following $E M R$, and complications including early/delayed bleeding and perforation.
\end{abstract}

Results Following service implementation, the number of EMRs rose from 11 in 2008 to 35 in 2011 , with the number of large polyps (>30 mm) rising from four in 2008 to 24 in 2011. Recurrent or residual adenomatous tissue fell from $75 \%$ in 2008 to $4.76 \%$ in 2011 . Only one perforation occurred over the 4 years $(0.8 \%$ perforation rate: 1 in 120 polypectomies). A reduction in surgical intervention for adenomatous polyp removal was observed during the audit period.

Conclusions Professional engagement and support by medical, surgical and nursing members of the endoscopy team promoted development of skill and confidence in EMR. Exposure to higher volumes of EMR procedures allowed successful removal of larger lesions, while maintained patient safety and reduced the need for surgical removal of benign polyps.

\section{Introduction}

Endoscopic management of benign colorectal polyps continues to develop. Polyps that were previously referred for surgical resection are now commonly removed endoscopically. Many centres have endoscopists that can remove large or flat polyps, but few have a formal endoscopic mucosal resection (EMR) service receiving most or all referrals. In 2007, as an initiative to optimise patient outcome from endoscopic removal of benign colonic polyps, the colorectal surgeons and gastroenterologists at the Queen Elizabeth Hospital, Gateshead, agreed that one endoscopist would specialise in these procedures. It was decided that large flat or sessile lesions identified by the medical and surgical endoscopic team would be referred for assessment and removal. The following article is a detailed description of how this service was instituted and subsequently developed over the next 4 years. Training and assessment of the endoscopist, the involvement of the multidisciplinary team (MDT), and changes to patient outcomes over time are discussed. The relevance of this service development to other district general hospitals, the wider question of who should be offering this type of service, and suggestions for appropriate quality indicators are considered.

\section{Background}

Injection-assisted EMR, often termed "saline-assisted" polypectomy, was first established in 1973 for flexible colonoscopy, ${ }^{1}$ and is now commonly used for large non-pedunculated colorectal polyps. The size, anatomical location and accessibility of a polyp relative to mucosal folds and flexures, determines the technical difficulty and risk of perforation and bleeding during removal. ${ }^{2-6}$ Adequate training, teamwork and quality assurance may lead to safe and high quality EMR.

Five levels of competency to remove colorectal polyps have been proposed. ${ }^{7}$ These vary from level 0 where no lesions are removed, and all referred to more 
skilled endoscopists, to level 4 where large flat lesions or other challenging polyps that may otherwise need removal surgically, are excised endoscopically. In the North-East of England there are several endoscopists with level 4 competency, but to date there is no formal tertiary referral service, therefore resulting in a shortfall of clinical provision in hospitals without a level 4 endoscopist.

\section{Setting up the EMR service} Training and assessment

Ideally endoscopists learning EMR should go through a formal training period composed of observation and assisting procedures, prior to performing the technique under direct observation, and finally independent practice. There is no formal programme such as this available in the UK. Mentorship is the training method that was used in Gateshead. An accredited Bowel Cancer Screening Programme (BCSP) colonoscopist from a nearby Institution, with level 4 polypectomy competency acted as mentor, and provided specific support, hands on guidance and assessment throughout the early period of training. This approach facilitated development of new skill within Gateshead, avoiding the need to refer patients to other hospitals for polypectomy. Level 3 and 4 polypectomies were done jointly, and formative assessments for specific polypectomy procedures were undertaken.

\section{New procedure committee and business case proposal}

EMR polypectomy was formally approved by the hospital New Procedure Committee. A business case was presented to the endoscopy managers for fortnightly therapeutic lists of two EMRs per list. Business managers and the Institution were therefore aware of the development of the service from the outset, specifically the need to reduce patient numbers on these lists to allow adequate time for the procedure, and the risks and benefits of the procedure.

\section{Quality assurance and MDT feedback}

Continual prospective audit of patient outcomes was undertaken, with annual presentation to the lower gastrointestinal (GI) MDT. The endoscopist provided a regular presence at the lower GI MDT, from where many of the new referrals came.

\section{Polyp referrals}

On referral receipt following discussion at the MDT or following referral from an endoscopist, a letter was dictated to patients explaining the EMR procedure, the risks involved, specifically the risk of perforation, and the alternative options including surgery. The letter was copied to the endoscopy unit and an appropriate duration of time allocated on an endoscopy list. The letter was also routinely copied to the referring endoscopist, and the consent process presented and discussed annually at the colorectal MDT. The need for timely referral to the EMR service from diagnostic endoscopy or CT scan was reinforced to prevent undue delay in consent letters being sent to patients.

The procedures outlined in this manuscript were those performed between 1 January 2008 and 31 December 2011 after referral by a medical or surgical endoscopist as part of the new EMR service. Lesions are also reported that were identified by the EMR endoscopist on a routine diagnostic list, and subsequently brought back for an EMR procedure after appropriate consent.

\section{Outcomes of the EMR service \\ Referrals}

Table 1 outlines the patients undergoing EMR from service establishment through the next 4 years. There was an approximate four-fold increase seen in patients receiving this procedure as part of the EMR service. Some patients had more than one polyp requiring EMR. The mean time from sending a consent letter to patients, and performing the index EMR procedure remained similar across all year groups at between $4-5$ weeks.

\section{Polyp size}

During the early stages of the EMR service, the majority of polyps were small, over $50 \%$ less than $20 \mathrm{~mm}$, and only a third over $30 \mathrm{~mm}$. As the service developed the proportion of larger polyps increased, such that by 2011 almost $85 \%$ of polyps were over $20 \mathrm{~mm}$, of which more than two thirds were over $30 \mathrm{~mm}$ in size.

Figure 1 demonstrates the absolute numbers of polyps removed per year according to polyp size, revealing a clear year on year increase in the number of larger polyps removed, particularly those $30 \mathrm{~mm}$ or more.

\section{Histology}

From 2009 onwards, there was a clear increase in polyps confirmed as either adenomatous or malignant after removal. Overall this histology accounted for between approximately $90 \%$ and $96 \%$ of polyp diagnoses. Polyps not identified as adenomatous or malignant were found to be inflammatory, hyperplastic or metaplastic.

Both the cancers identified in 2008 and 2009 showed invasion up to the resection margins. However, the four cancers removed by EMR in 2010 and 2011 were completely cleared with no evidence of local invasion, lymphadenopathy or metastasis on colonic resection or subsequent imaging. None of the attempted or removed polyps were known to be cancers prior to EMR, all were thought to be adenomatous.

\section{Recurrence}

In the EMR service, recurrence is assessed endoscopically at 3 months following index EMR in those patients identified as having adenomas. Photos are routinely taken of the polypectomy scar to prove 
Table 1 Polyp size, histology and 3 month recurrence for patients undergoing endoscopic mucosal resection (EMR) between 2008 and 2011

\begin{tabular}{|c|c|c|c|c|}
\hline & 2008 & 2009 & 2010 & 2011 \\
\hline Patients undergoing EMR (n) & 11 & 23 & 48 & 33 \\
\hline EMRs performed $(n)^{*}$ & 11 & 26 & 48 & 35 \\
\hline Mean time from consent letter to index EMR in days (range) & $34.25(15-48)$ & $30.29(4-56)$ & $31.57(4-80)$ & $35.58(2-169)$ \\
\hline \multicolumn{5}{|l|}{ Relative size of polyps } \\
\hline$<20 \mathrm{~mm}(\%)$ & 54.55 & 50 & 35.42 & 17.14 \\
\hline $20-29 \mathrm{~mm}(\%)$ & 9.09 & 11.54 & 27.08 & 25.71 \\
\hline$\geq 30 \mathrm{~mm}(\%)$ & 36.36 & 38.46 & 37.5 & 57.14 \\
\hline Range of polyp size (mm) & $8-80$ & $10-80$ & $4-120$ & $10-60$ \\
\hline \multicolumn{5}{|l|}{ Ultimate histology } \\
\hline Adenoma: n (\%) & $8 / 11(72.73)$ & $24 / 26(92.31)$ & $40 / 48(83.33)$ & $32 / 35(91.43)$ \\
\hline Malignancy: n (\%) & $1 / 11(9.09)$ & $1 / 26(3.85)$ & $3 / 48(6.25)$ & $1 / 35(2.86)$ \\
\hline Hyperplastic/metaplastic/inflammatory: n (\%) & $2 / 11(18.18)$ & $1 / 26(3.85)$ & $5 / 48(10.42)$ & $2 / 35(5.71)$ \\
\hline \multicolumn{5}{|l|}{ Polyp recurrence at 3 month endoscopy: $n(\%)$} \\
\hline Overall & $6 / 8(75.00)$ & $7 / 15(46.7)$ & $8 / 36(22.22)$ & $1 / 21(4.76)$ \\
\hline$<20$ mm (n) & 2 & 2 & 1 & \\
\hline $20-29 \mathrm{~mm}(\mathrm{n})$ & & 1 & 3 & \\
\hline$\geq 30 \mathrm{~mm}(\mathrm{n})$ & 4 & 4 & 4 & 1 \\
\hline
\end{tabular}

${ }^{\star} \mathrm{A}$ small number of patients had more than one polyp requiring EMR.

clearance. If any residual islands of adenoma are seen, these are biopsied, and either raised and snared if possible, or destroyed with Argon Plasma Coagulation. Reasons for not performing EMR at 3 months include the finding of non-adenomatous histology or malignancy at index EMR, intercurrent co-morbidity making EMR higher risk, or patient preference not to undergo a repeat procedure.

As demonstrated in table 1 , in 2008 , the adenoma recurrence at 3 months was $75 \%(n=6 / 8)$, however this subsequently fell to $46.7 \%(\mathrm{n}=7 / 15)$ in $2009,22.22 \%$ $(n=8 / 36)$ in 2010, and 4.76\% $(n=1 / 21)$ in 2011. Statistics for 2010 and 2011 are therefore comparable with other published series, which describe a recurrence rate of between $1.7-28 \%$ at follow up examination. ${ }^{8-12}$

\section{Complications}

The hospital Patient Administration System was interrogated to identify the 30-day re-admission rate

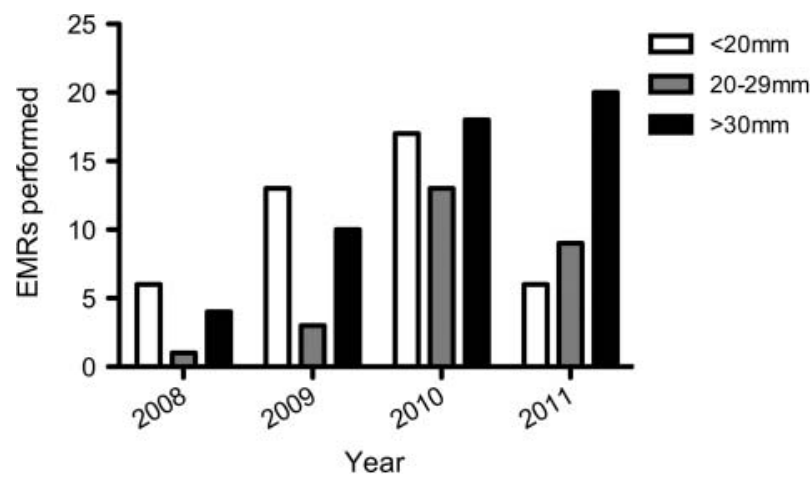

Figure 1 Number of endoscopic mucosal resections performed per year, stratified according to polyp size. following EMR, and notes were reviewed to establish the cause of re-admission. Complications were characterised as immediate peri-procedural bleeding that required therapy (eg, endoclips or adrenaline), delayed bleeding leading to re-admission, postpolypectomy syndrome leading to re-admission, perforation, and death. A summary of complications is presented in table 2.

In 2008 there was one immediate bleed following a $25 \mathrm{~mm}$ polyp removal, controlled with adrenaline injection and endoclips. The patient was admitted for 3 days observation then discharged. The day following discharge the patient experienced a cardiac arrest and died. Post mortem examination identified a perforation at the polypectomy site. A root cause analysis investigation performed concluded that the death was related to the polypectomy, with a delayed perforation.

In 2009 there was one delayed bleed 7 days following removal of a $40 \mathrm{~mm}$ rectal polyp. This required

Table 2 Summary of annual endoscopic mucosal resection complications

\begin{tabular}{|c|c|c|c|c|}
\hline & $\begin{array}{c}2008 \\
(n=11)\end{array}$ & $\begin{array}{c}2009 \\
(n=26)\end{array}$ & $\begin{array}{c}2010 \\
(n=48)\end{array}$ & $\begin{array}{r}2011 \\
(n=35)\end{array}$ \\
\hline $\begin{array}{l}\text { Post polypectomy } \\
\text { syndrome requiring } \\
\text { re-admission: } n(\%)\end{array}$ & - & - & $1(2.08)$ & - \\
\hline Immediate bleed: $\mathrm{n}(\%)$ & $1(9.09)$ & - & $2(4.17)$ & - \\
\hline $\begin{array}{l}\text { Delayed bleed requiring } \\
\text { re-admission: } \mathrm{n}(\%)\end{array}$ & - & $1(3.85)$ & $3(6.25)$ & $1(2.86)$ \\
\hline Perforation: n (\%) & $1(9.09)$ & - & - & - \\
\hline Death: $n(\%)$ & $1(9.09)$ & - & - & - \\
\hline
\end{tabular}


re-admission and was managed successfully with adrenaline, injection and endoclips.

In 2010 there were two peri-procedural bleeds after a $20 \mathrm{~mm}$ and $40 \mathrm{~mm}$ polypectomy respectively, both responded to endoscopic therapy without further need for intervention. Both were admitted overnight for observation. One received adrenaline and endoclips, the other adrenaline and heater probe therapy. There were three delayed bleeds, two of which were in patients with polyps greater than $30 \mathrm{~mm}$. One patient was admitted, re-endoscoped and treated successfully with adrenaline and heater probe therapy. The other two patients were admitted for observation, but no intervention was necessary. One patient was admitted the same day as the EMR with severe abdominal pain and peritonism. A CT scan showed no evidence of perforation, they were commenced on intravenous antibiotics and the pain settled. The patient was discharged $48 \mathrm{~h}$ later with a presumed diagnosis of post polypectomy syndrome.

There was only one complication in 2011. This was a delayed bleed, requiring a two unit blood transfusion but no endoscopic therapy.

Overall bleeding rates requiring admission are comparable with published series, which describe a risk of between $2.9 \%$ and $16 \% .{ }^{5-11} 13$

The overall perforation rate for the 4 years was $0.8 \%$ ( 1 in 120 polypectomies).

\section{Polyps not attempted or not completed}

In addition to the EMRs outlined in table 1, each year, there were a small number of patients in which the procedure was either not attempted or not completed.

There were three cases overall where after consideration of the risks and benefits of the procedure, the patients decided not to proceed to EMR (two in 2010, one in 2011). In all cases the patient also declined surgery.

In three cases the endoscopist decided not to attempt EMR (two in 2010, one in 2011). In two of these, therapeutic endoscopy was not attempted due to an adverse risk:benefit ratio as a consequence of significant co-morbidity (advanced liver disease and dementia respectively). The other procedure was not attempted, as at endoscopy the lesion was complex and extensively involved the caecum and ileo-caecal valve. The patient in this circumstance was referred on for surgery.

Over the 4 years there were a total of nine incomplete polypectomies, where the endoscopist was technically unable to clear all the polyp tissue at EMR (two in each of 2008-2010, three in 2011). Five were due to non-lifting of the polyp on injection, precluding successful snare, and in the remaining four patients complete removal was unsuccessful after prolonged de-bulking. In total six of these nine polyps were found to be malignant. Five were primary colonic cancers, and one a metastatic deposit from an ovarian primary.

\section{Reduction in surgical resection rates}

An audit of surgery for benign colonic lesions analysed all resection specimens in 2005 and 2010. Six resections for adenomatous colorectal lesions were performed in 2005. None had attempted EMR and lesions varied from one that would have been technically challenging (a carpet like lesion in the caecum, $80 \times 65 \mathrm{~mm}$ measured at resection) and five lesions that would now be routinely attempted endoscopically ( $25 \mathrm{~mm}$ sessile caecal, $45 \mathrm{~mm}$ sessile rectal, $65 \mathrm{~mm}$ pedunculated sigmoid, $20 \mathrm{~mm}$ sessile rectal, $20 \mathrm{~mm}$ sessile caecal polyps respectively). In 2010 there were three resections for adenomatous colorectal lesions. One was technically challenging covering the caecum and ileocaecal valve and referred for surgery (after EMR assessment) as described above. One was not referred to the EMR service $(60 \mathrm{~mm}$ pedunculated ascending) and the other was assessed as not removable by one of the other gastroenterologists as it did not rise with injection.

\section{Discussion}

The six gastroenterologists at Gateshead work collaboratively. All undertake 'core' gastroenterology provision including upper and lower GI endoscopic therapy; however, all also have areas of specialist interest. The team was supportive of one endoscopist setting up a service to provide EMR, enabling one operator to gain the experience of increasing numbers of larger polyps. Subsequently the other gastroenterologists no longer routinely undertake EMR of large polyps. The data presented in this manuscript demonstrates that over a 4-year period as the new service became established in the Organisation, the number of procedures performed by this service increased fourfold and was associated with a reduction in recurrent/residual adenomatous tissue from $75 \%$ in the 1 st year, to $4.76 \%$ in the 4 th year. This was coupled with an overall reduction in the incidence of serious complications including perforation, immediate and delayed bleeding. Resection rates for benign disease have fallen since service introduction, and the surgical approach to removal of benign lesions has undoubtedly changed during this time, with EMR now the preferred first option. Consequently, this suggests a significant improvement in patient outcome as a result of the service development. This also suggests that increased therapeutic experience, initially of approximately 50 procedures and higher annual procedure volumes of at least 30 procedures, leads to better patient outcome.

Based on the Gateshead experience, box 1 outlines suggestions for setting up a formalised EMR service in a district general hospital. Crucial to implementation of this type of service is training and ongoing support 
Box 1 Suggestions for setting up an endoscopic mucosal resection (EMR) service in a district general hospital

- Depending on annual EMR volume, identify one or two endoscopists willing to develop the necessary skills to introduce and maintain the service. Two endoscopists may be preferable to facilitate continuous service without interruption for leave periods, and provides an extra experienced pair of hands for complex procedures. Alternatively endoscopists from neighbouring Institutions can pair with one another to provide this type of service across two or more hospitals.

- Gain support from endoscopic colleagues and the lower GI multidisciplinary team (MDT).

- A business case helps to justify EMR lists to endoscopy managers, and allows enough time for complex procedures.

- Prepare the patient, the Institution and yourself for risk of perforation and bleeding. Perforation should be expected in $1 \%$ of EMR polypectomies and can often be managed endoscopically.

- Early procedures should be undertaken with the supervision of an experienced, ideally level four polypectomy competent endoscopist.

- At least 30 procedures per year should be performed per endoscopist to maintain competency.

- Regular formal assessment of technique should be undertaken using DOPyS polypectomy assessment score sheet.

- Perform regular audit and present results to the Lower GI MDT annually.

- Be flexible and responsive to referrals ensuring timely index EMR and consistent follow up and discharge.

- Always attend Lower GI MDT and liaise with other 'EMR colleagues' in the region on complex cases.

from a more experienced endoscopist. Assessment, feedback and reflection is as useful in learning more advanced techniques such as EMR polypectomy, as it is when learning the basics of colonoscopy. The recently developed and validated Direct Observation of Polypectomy Skills (DOPyS) proforma may allow objective assessment of skills development. ${ }^{14}$

Recurrent or residual adenomatous tissue is more difficult to remove after a prior attempt at polypectomy due to fibrosis, and so clearance at first EMR is desirable. ${ }^{9}$ In the Gateshead series, all but one polyp were cleared by further snare and diathermy on 2nd or subsequent attempts. The reduction in 3-month adenoma recurrence seen in Gateshead suggests one endoscopist performing this procedure in a nonspecialist district general hospital is preferable to many endoscopists performing EMR; however, depending on the workload of the hospital and to ensure cover during periods of annual leave it may be

Box 2 Considerations for quality indicators when setting up an endoscopic mucosal resection (EMR) service

- Time to index EMR from consent letter or diagnostic procedure.

- Appropriate consent outlining higher risk of complication than standard colonoscopy, including risk of perforation, bleeding and pain.

- Incidence of complications.

- Recurrence/residual adenomatous tissue at 3 months.

- Continuous or at least 6 monthly audit of service and patient outcomes.

\section{Box 3 Technical tips}

- Book adequate time/avoid over-booking lists especially when starting a service off-Full clearance at first polypectomy session is desirable.

- Maximise access and visualisation with position change/short scope/buscopan/washing (suction nearby pools in case of perforation).

- Use dye to raise polyps (clarifying margins) and to enhance pit pattern identification.

- Increase 'bite size' at your own pace. Avoid worrying about en bloc resection rate early (almost all recurrence/residual can be managed-perforation can be fatal).

- Start small and take on bigger polyps gradually. Refer more complex polyps to experienced colleagues and see how they do it.

- Snares have their own technical characteristics which take time to master. Get used to one snare-it can be made to do most things. Small snares are useful for limited access and a stiff snare for 'slippery' flat lesions.

advisable for two endoscopists to take responsibility for such a service. Although adenomatous polyp recurrence is a clear marker of outcome following EMR, the data presented suggests other quality indicators when establishing an EMR service. These are described in box 2 .

Data from the Gateshead EMR service highlights that training in EMR takes several years after independence in diagnostic colonoscopy. Consequently it is unlikely that the annual number of cases for the average district general hospital is large enough to allow training of registrars, and maintenance of skills for a dedicated consultant. Development of EMR skills as part of registrar training may therefore only be appropriate in large centres, with sufficient training opportunities to allow clear progression of skills.

Which endoscopists should be providing an EMR service is unclear. It could be argued that only BCSP endoscopists should be performing these procedures, as they now undertake formal assessment of polypectomy technique and large polyps are detected by the BCSP. However in Gateshead, 95\% of colonoscopies are performed within the symptomatic routine endoscopy service, and so there is a significant chance of identifying polyps requiring EMR in this population, as outlined in this manuscript. With appropriate training, and experience, non-BCSP endoscopists can successfully provide a safe and effective service. Box 3 outlines some technical tips learned as a result of the development of the EMR service at Gateshead.

\section{Conclusions}

EMR is the treatment of choice for most large benign adenomatous polyps in the colon. Endoscopic skill to remove these larger polyps takes time to develop and recurrence rate is a good quality indicator of how this skill improves. Supportive colleagues, a supportive management structure and above all good trainers and mentors are essential for a service to develop and flourish. 


\section{What is already known on this topic}

EMR is an established therapeutic procedure to remove large sessile or flat adenomatous lesions within the colon.

\section{What this study adds}

- This study demonstrates that the development of a high quality EMR service can be achieved in a district general hospital, producing patient outcomes comparable to published series.

- This was achieved by collaborative working between physician, surgeon and nursing colleagues to support the training of, and subsequent referral to a designated therapeutic endoscopist whom would specialise in this technique.

How this study might impact on clinical practice in the foreseeable future

The training model and prospective assessment of outcomes described in this paper may be employed by other Institutions to deliver similar high quality EMR services.

Acknowledgements The authors thank Dr John Painter for his direct supervision and mentorship during the establishment of the Gateshead EMR service. Thanks also to Dr Matt Rutter, and the late Dr Ken Mathewson for their training in EMR. The authors are grateful to Dr Matt Rutter and Dr Roland Valori for their comments during the preparation of this manuscript.

Contributors JAB was the endoscopist and carried out prospective audit of service development. CAL was responsible for data analysis. Both authors contributed to manuscript drafting.

Competing interests None.

Ethics approval This manuscript describes the findings of prospective audit carried out during a service development exercise. Ethical approval was therefore not required.

Provenance and peer review Not commissioned; externally peer reviewed.

\section{References}

1. Deyhle P, Jenny S, Fumagalli I. Endoscopic polypectomy in the proximal colon. A diagnostic, therapeutic (and preventive?) intervention. Dtsch Med Wochenschr 1973;98:219-20.

2. Gallegos-Orozco JF, Gurudu SR. Complex colon polypectomy. Gastroenterol Hepatol (N Y) 2010;6:375-82.

3. Monkemuller K, Neumann H, Malfertheiner P, et al. Advanced colon polypectomy. Clin Gastroenterol Hepatol 2009;7: 641-52.

4. Kim HS, Kim TI, Kim WH, et al. Risk factors for immediate postpolypectomy bleeding of the colon: a multicenter study. Am J Gastroenterol 2006;101:1333-41.

5. Metz AJ, Bourke MJ, Moss A, et al. Factors that predict bleeding following endoscopic mucosal resection of large colonic lesions. Endoscopy 2011;43:506-11.

6. Dafnis G, Ekbom A, Pahlman L, et al. Complications of diagnostic and therapeutic colonoscopy within a defined population in Sweden. Gastrointest Endosc 2001;54:302-9.

7. Segnan N, Patrick J, Karsa von L. European guidelines for quality assurance in colorectal cancer screening and diagnosis. 1st edn. International Agency for Research on Cancer, Luxemberg, Publications Office of the European Union, 2010.

8. Stergiou N, Riphaus A, Lange P, et al. Endoscopic snare resection of large colonic polyps: how far can we go? Int $J$ Colorectal Dis 2003;18:131-5.

9. Moss A, Bourke MJ, Williams SJ, et al. Endoscopic mucosal resection outcomes and prediction of submucosal cancer from advanced colonic mucosal neoplasia. Gastroenterology 2011;140:1909-18.

10. Tanaka S, Haruma K, Oka S, et al. Clinicopathologic features and endoscopic treatment of superficially spreading colorectal neoplasms larger than $20 \mathrm{~mm}$. Gastrointest Endosc 2001;54:62-6.

11. Lee SH, Park JH, Park do H, et al. Clinical efficacy of EMR with submucosal injection of a fibrinogen mixture: a prospective randomized trial. Gastrointest Endosc 2006;64:691-6.

12. Arebi N, Swain D, Suzuki N, et al. Endoscopic mucosal resection of 161 cases of large sessile or flat colorectal polyps. Scand J Gastroenterol 2007;42:859-66.

13. Iishi $\mathrm{H}$, Tatsuta $\mathrm{M}$, Iseki $\mathrm{K}$, et al. Endoscopic piecemeal resection with submucosal saline injection of large sessile colorectal polyps. Gastrointest Endosc 2000; 51:697-700.

14. Gupta S, Anderson J, Bhandari P, et al. Development and validation of a novel method for assessing competency in polypectomy: direct observation of polypectomy skills. Gastrointest Endosc 2011;73:1232-9 e2. 